\title{
Marco conceptual para la discusión sobre el modelo de Diseño Instruccional en Educación a Distancia en la Universidad Nacional Abierta y a Distancia
}

Arellys de Jesús Correa Rodríguez ${ }^{1}$

Sonia Mayerly Castro Bedoya ${ }^{2}$

\begin{abstract}
Resumen
La finalidad de esta investigación es proporcionar un marco conceptual para la discusión sobre el modelo de Diseño Instruccional (DI) en Educación a Distancia en la Universidad Nacional Abierta y a Distancia. Inicialmente se realizó una revisión de la literatura de investigaciones asociadas al Diseño Instruccional durante los últimos cinco años, las cuales coinciden con la importancia del Diseño Instruccional en la Educación a Distancia. En segunda instancia se realizó un estudio documental dividido en dos fases: la primera basada en recolección de información y su organización en fichas de trabajo y la segunda fase dedicada al análisis de la información utilizando una matriz analítica y un cuadro comparativo entre un modelo de D.I. Dick Carey y Carey y el estándar CORE de la UNAD para proveer una información organizada y clara. Finalmente de acuerdo a los hallazgos obtenidos en la recopilación y análisis de la información para identificar el modelo de diseño instruccional utilizado en la UNAD, se establecen algunas conclusiones y recomendaciones a tener en cuenta para el diseño de cursos virtuales.
\end{abstract}

2 Psicóloga Candidata a Master of Arts in Education Specialization in Online Education en UNAD - Florida. Universidad Nacional Abierta y a Distancia - UNAD Florida Cargo Tutora de la Escuela de Ciencias Sociales Artes y Humanidades Bogotá - Cundinamarca - Colombia maye.26@hotmail.com 
Palabras claves: campus virtual, diseño instruccional, educación a distancia, educación virtual.

\title{
A conceptual framework for discussing an instructional design model for distance education at UNAD
}

\begin{abstract}
This research intends to provide a conceptual framework to discuss the DI model of Instructional Design in Distance Education at the Open and Distance National University (UNAD).

Initially, a literature review on research associated to instructional design during the last five years performed, all of which agree on the importance of instructional design in distance education. Secondly a documentary study was conducted in two phases: the first stage was based on gathering information and organizing data in worksheets, and the second stage focused on the analysis of information using an analytical matrix and a comparative table between Dick Carey \& Carey's model and the standard UNAD's CORE in order to deliver clear and organized information. Finally, several conclusions and recommendations to be taken into account when designing online courses are made based on the findings from the gathering and analysis of information in order to identify the instructional design model used at UNAD.
\end{abstract}

Keywords: Virtual campus, instructional design, distance education, online education.

Recibido: 14 de julio 2011 Aceptado: 28 de octubre 2011

\section{Introducción}

Actualmente la educación a distancia ha tenido gran acogida debido a que se apoya en el uso de la Internet como medio para obtener aprendizaje y 
rompe con los esquemas de los modelos tradicionales que limitan a las personas a estudiar en una institución presencial. Sin embargo, no basta con desarrollar cursos en línea a través de una plataforma tecnológica. Éste es un proceso que requiere de planeación, diseño, implementación y evaluación teniendo en cuenta las teorías de aprendizaje. En este sentido, el Diseño Instruccional (DI) como proceso consiste en el desarrollo sistemático de los elementos instruccionales, usando las teorías del aprendizaje y las teorías instruccionales para asegurar la calidad de la instrucción. Esto incluye el análisis de necesidades de aprendizaje, las metas y el desarrollo materiales y actividades instruccionales, la evaluación del aprendizaje y el seguimiento (Berger y Kam, 1996).

Dado que no se puede concebir la Educación a Distancia igual que la Educación Tradicional es imperioso para la planeación de un curso en línea tener en cuenta las características de los estudiantes, determinar qué es lo que deben aprender, cómo se realizará dicho proceso de enseñanza-aprendizaje y saber si al final de la instrucción se alcanzó la meta de aprendizaje. Con base en esto la instrucción se seguirá implementando, se modificará o cambiará completamente con el fin de garantizar la calidad educativa. La presente investigación está motivada por la necesidad de conocer si existe un modelo de Diseño Instruccional para la realización de cursos virtuales en la UNAD, ya que no se evidencia un documento que describa algún modelo de este tipo implementado en la universidad.

\section{Metodología}

Para la Universidad Nacional Abierta y a Distancia -UNAD - la innovación y la incorporación tecnológica son elementos esenciales en los procesos institucionales para garantizar la calidad en la educación. Por tal motivo, la UNAD hace uso de un campus virtual a través del cual se ofertan cursos por mediación virtual. El campus está diseñado en la plataforma tecnológica Moodle, la cual es un sistema de gestión de cursos libres que ayuda al proceso de aprendizaje en línea, basado en el constructivismo y en el aprendizaje colaborativo. (Salazar et al., 2008).

La UNAD diseña en Moodle los cursos virtuales basándose en el estándar CORE, un dispositivo del orden tecno- pedagógico, curricular y didáctico digital diseñado para generar aprendizaje autónomo mediante la estructuración de procesos sistémicos de formación en el marco de la modalidad de Educación Abierta y a Distancia. Sin embargo, la comunidad académica ha manifestado que la actual estructura de los cursos virtuales limitan tanto al tutor como al estudiante al momento de realizar los proceso de enseñanza-aprendizaje. El tutor no tiene incidencia en el diseño de las actividades, su labor es más de orientación hacia el estudiante y este utiliza los recursos de comunicación en campus para solicitar nuevas oportunidades a la hora de entregar actividades y no para la producción de su conocimiento. Los foros académicos son utilizados para la entregas de trabajos 
pero no se presentan dentro de ellos debates sobre un determinado tema, por tanto el estudiante se limita a enviar una actividad y el tutor a calificar. Teniendo en cuenta esto, existe la necesidad de fortalecer el carácter pedagógico de las actividades así como los contenidos didácticos de los cursos. Hasta la fecha no existe un documento que describa el modelo de Diseño Instruccional utilizado en los cursos virtuales de la UNAD. Así, aunque en los cursos es evidente la influencia de diferentes modelos como el de Dick Carey y Carey y ASSURE, donde el primero está orientados hacia el sistema y el último hacia el salón de clases, lo que existe no son más que supuestos que deben ser confirmados y documentados.

La instrucción se concibe no sólo como un proceso, sino como un sistema que comprende un conjunto de procesos interrelacionados: análisis, diseño, producción, desarrollo (implementación) y evaluación. La relación entre esos procesos debe ser sistémica más que sistemática, ya que estos pueden ocurrir en paralelo, simultáneamente yen estrecha interrelación -antes que de manera lineal-. Por lo tanto, es necesario conocer cuál es el modelo de Diseño Instruccional utilizado en la universidad Nacional Abierta y a Distancia.

Dado que no se conoce un documento que describa el modelo de Diseño Instruccional utilizado en la Universidad, y teniendo en cuenta lo manifestado por la comunidad académica en relación a las limitaciones en cursos virtuales, es necesario conocer y analizar cuál es el modelo de Diseño Instruccional utilizado en la UNAD para identificar las fortalezas y debilidades del modelo. El Diseño Instruccional de un curso debe ser visto como todo un sistema y una relación entre sus componentes: instructor, estudiante, materiales y tecnología (Simonson et al., 2006).

\section{Tipo de estudio}

De acuerdo con la documentación proporcionada por la universidad y teniendo en cuenta algunos de los modelos de Diseño Instruccional existentes se desarrolló un estudio documental descriptivo para determinar cuál es el modelo de Diseño Instruccional que se utiliza en los cursos virtuales de la UNAD. La investigación descriptiva documental aborda fenómenos que no pueden ser explicados a través de números o índices, sino que son analizados como sistemas complejos e interrelacionados desde el punto de vista humano. Se utiliza entonces la descripción de los hechos en la generación del conocimiento y la comprensión de los fenómenos.

\section{Resultados y discusión}

La documentación estuvo centrada en la información suministrada por la Vicerrectoría de Medios y Mediaciones Pedagógicas (VIMMEP), que es un componente estructural del sistema organizacional de la Universidad. Con un 
carácter misional, la VIMMEP es responsable de dirigir, diseñar, producir y administrar los medios y mediaciones pedagógicas propias de la educación abierta y a distancia como son:

Salazar, et al. (2008). Curso de diseño y administración de aulas virtuales en el campus virtual de la UNAD. Bogotá: GIDT (Gerencia de Innovación y Desarrollo Tecnológico) - Universidad Nacional Abierta y a Distancia.

Acuerdo: Por el cual se establecen las condiciones pedagógicas y tecno didácticas para el diseño y acreditación de los cursos académicos sobre las cuales centra sus actividades de aprendizaje la Universidad Nacional Abierta y a Distancia, UNAD.

Ponencia en video sobre la arqueología de la educación a Distancia en la UNAD a cargo del Dr. Salazar R. 2011, Freddy Rodríguez (2009). Modulo de Competencias comunicativas de la UNAD

De igual forma Se revisan dos (2) modelos de diseño instruccional uno orientados al sistema y otro al salón de clases así: Modelo de Dick Carey y Carey (orientado al sistema) y el modelo de ASSURE (Orientado al salón de clases).

Elaboración de fichas de trabajo: son los instrumentos en los que se registra de manera independiente los datos de las obras consultadas. Estas fichas contienen todos los datos básicos de un documento: autores, título, edición, año y lugar de publicación, colección y número. En este estudio se utilizó la ficha de resumen para el registro de los de los documentos que pudieran proporcionar información relacionada con el modelo de Diseño Instruccional utilizado en la UNAD.

Método de análisis de datos: Después de la realización de las fichas de trabajo se utilizó una matriz analítica para proveer una información organizada y clara de los documentos recolectados. También se estableció un paralelo entre el modelo Dick Carey y Carey y el estándar CORE de la UNAD.

Para dar respuesta a la pregunta de investigación que busca indagar qué modelo de Diseño Instruccional utiliza la Universidad Nacional Abierta y a Distancia en los cursos de campus virtual,, los resultados se visualizan en las siguientes tablas:

Tabla 1. Matriz análisis de la Información

\begin{tabular}{|l|l|l|l|}
\hline FUENTE & AUTOR & AÑO & ANÁLISIS \\
\hline $\begin{array}{l}\text { Modulo de } \\
\text { Competencias } \\
\text { comunicativas } \\
\text { UNAD }\end{array}$ & $\begin{array}{l}\text { F. Fredy } \\
\text { Rodríguez }\end{array}$ & 2009 & $\begin{array}{l}\text { La apropiación del modelo ASSURE para el di- } \\
\text { seño del curso de competencias comunicativas } \\
\text { contempla el análisis inicial de los posibles parti- } \\
\text { cipantes. Conocer previamente las características } \\
\text { de los estudiantes nos permite orientar los conte- } \\
\text { nidos con el fin de satisfacer las necesidades más } \\
\text { apremiantes. }\end{array}$ \\
\hline
\end{tabular}


Marco conceptual para la discusión sobre el modelo de Diseño Instruccional en Educación a Distancia en la Universidad Nacional Abierta y a Distancia, artículo producto de la investigación

\begin{tabular}{|c|c|c|c|}
\hline FUENTE & AUTOR & AÑO & ANÁLISIS \\
\hline $\begin{array}{l}\text { Instructional Media } \\
\text { and Technologies } \\
\text { for Learning }\end{array}$ & $\begin{array}{l}\text { Sharon,Heinich, } \\
\text { Russell } \\
\text { Smaldino, } \\
\text { Robert, James D } \\
\text { Robert. }\end{array}$ & 2002 & $\begin{array}{l}\text { EL modelo ASSURE es un (Sistema de Diseño } \\
\text { Instruccional) proceso que fue modificado para ser } \\
\text { usado por los maestros un en salón de clases. El } \\
\text { proceso ISD es aquel que los maestros y los capa- } \\
\text { citadores pueden usar para diseñar y desarrollar } \\
\text { el ambiente de aprendizaje más apropiado para } \\
\text { sus estudiantes. Este proceso se puede utilizar } \\
\text { para planear las lecciones así como para mejo- } \\
\text { rar la enseñanza y el aprendizaje centrado en el } \\
\text { estudiante. }\end{array}$ \\
\hline $\begin{array}{l}\text { The Systematic } \\
\text { Design of } \\
\text { Instructions }\end{array}$ & $\begin{array}{l}\text { Walter Dick, Lou } \\
\text { Carey, James O. }\end{array}$ & 2005 & $\begin{array}{l}\text { El modelo de diseño instruccional de Dick \& Ca- } \\
\text { rey y Carey es uno de los más utilizados en el } \\
\text { ámbito educativo, se define como: "un sistema } \\
\text { compuesto por fases o componentes que inte- } \\
\text { ractúan entre sí; Este modelo está orientado } \\
\text { a los sistemas para el diseño de la instrucción, } \\
\text { está compuesto por diez fases las cuales son: } \\
\text { Identificación de la meta instruccional, análisis de } \\
\text { la meta instruccional, análisis de los estudiantes } \\
\text { y del contexto, redacción de objetivos, desarrollo } \\
\text { de instrumentos de evaluación, elaboración de la } \\
\text { estrategia instruccional, desarrollo y selección de } \\
\text { materiales de instrucción, diseño y desarrollo de } \\
\text { evaluación formativa diseño y desarrollo de eva- } \\
\text { luación sumativa y revisión de la instrucción. }\end{array}$ \\
\hline $\begin{array}{l}\text { Curso de Diseño y } \\
\text { Administración en el } \\
\text { campus virtual de la } \\
\text { UNAD }\end{array}$ & $\begin{array}{l}\text { Grupo de } \\
\text { innovaciones } \\
\text { pedagógicas }\end{array}$ & 2008 & $\begin{array}{l}\text { El estándar CORE es eje central de las activida- } \\
\text { des de aula en los cursos académicos a través de } \\
\text { la mediación virtual, establece como deben ser } \\
\text { los contenidos dentro de los cursos y Presenta el } \\
\text { proyecto metodológico y evaluativo en los cursos } \\
\text { virtuales a por medio de tres fases: } \\
\text { Reconocimiento: busca identificar conocimien- } \\
\text { tos previos en el estudiante. } \\
\text { Profundización: donde el estudiante se apropia } \\
\text { de los conceptos propios del curso, de desarrolla } \\
\text { por medio de lecciones evaluativas un quiz de la } \\
\text { unidad. } \\
\text { Transferencia: Es la aplicación de los conoci- } \\
\text { mientos obtenidos en la fase anterior y se desa- } \\
\text { rrolla a través de los trabajos colaborativos. }\end{array}$ \\
\hline $\begin{array}{l}\text { Acuerdo por el cual } \\
\text { se establecen las } \\
\text { condiciones pedagó- } \\
\text { gicas y tecno-didác- } \\
\text { ticas para el diseño } \\
\text { y acreditación de los } \\
\text { cursos académicos } \\
\text { sobre las cuales } \\
\text { centra sus activida- } \\
\text { des de aprendizaje } \\
\text { la Universidad } \\
\text { Nacional Abierta y a } \\
\text { Distancia, UNAD }\end{array}$ & $\begin{array}{l}\text { El Consejo } \\
\text { Académico de } \\
\text { la Universidad } \\
\text { Nacional Abierta } \\
\text { y a Distancia } \\
\text { UNAD, }\end{array}$ & 2010 & $\begin{array}{l}\text { Establece que el CORE se ha instituido desde } \\
\text { el segundo semestre de } 2006 \text { como el disposi- } \\
\text { tivo académico, pedagógico, curricular y didác- } \\
\text { tico mediante el cual se diseña, virtualiza y se } \\
\text { ofrece el conjunto de cursos académicos que } \\
\text { sirve de base al desarrollo de los procesos for- } \\
\text { mativos que adelanta la institución. Los objetivos } \\
\text { del CORE son: Facilitar el diseño de procesos de } \\
\text { aprendizaje, fomentar los lineamientos, estánda- } \\
\text { res y protocolos tecno pedagógicos y didácticos, } \\
\text { establecer las condiciones tecno pedagógicas } \\
\text { básicas para la selección de estrategias tecno } \\
\text { didácticas. }\end{array}$ \\
\hline
\end{tabular}




\begin{tabular}{|l|l|l|l|}
\hline FUENTE & AUTOR & AÑO & ANÁLISIS \\
\hline $\begin{array}{l}\text { Video Arqueología } \\
\text { de la educación } \\
\text { a Distancia en la } \\
\text { UNAD }\end{array}$ & Salazar R. & 2011 & $\begin{array}{l}\text { Inicia indicando que al principio el modelo ins- } \\
\text { truccional está centrado en la enseñanza, sin } \\
\text { embargo no menciona ningún modelo instruc- } \\
\text { cional. Los medios se conjugan en los diseños } \\
\text { instruccionales para potencializar el aprendizaje } \\
\text { autónomo. Se establecen tres momentos de la } \\
\text { educación a Distancia en la UNAD, el primero } \\
\text { llamado modelo industrializado (1982 - 1987) } \\
\text { donde la universidad tenía contendidos con ac- } \\
\text { tividades distribuidos basado en tecnología edu- } \\
\text { cativa, las actividades reiteran lo que es sustanti- } \\
\text { vo para los estudiante los contenidos son lo más } \\
\text { importante en el estudiante. } \\
\text { En el modelo artesanal (1988-2004) lo peda- } \\
\text { gógico pasa a un segundo plano, el tutor asume } \\
\text { el liderazgo en la transmisión del conocimiento } \\
\text { en las aulas, la universidad se vuelve semi-pre- } \\
\text { sencial y de manera intensiva los fines de sema- } \\
\text { na. A partir del 2005 la UNAD ha venido incur- } \\
\text { sionando en el modelo virtual se ha alimentado } \\
\text { de las mediaciones y de los cursos e-learning, } \\
\text { las actividades son el centro del desarrollo de } \\
\text { los procesos formativos y los contenidos son } \\
\text { aquellos que permiten el desarrollo de las ac- } \\
\text { tividades, y son contendidos distribuidos, como } \\
\text { ejemplo el diseño instruccional del e-learning } \\
\text { donde las actividades son el centro de los proce- } \\
\text { so de formación }\end{array}$ \\
\hline & & \\
\hline
\end{tabular}

Tabla 2. Cuadro Comparativo Modelo Dick Carey y Carey y estándar CORE

\begin{tabular}{|l|l|}
\hline MODELO DICK, CAREY Y CAREY & ESTÁNDAR CORE UNAD CAREY \\
\hline Identificación de la meta Instruccional & $\begin{array}{l}\text { Competencia general de aprendizaje se en- } \\
\text { cuentra dentro del protocolo del curso. }\end{array}$ \\
\hline Análisis de la Instrucción & $\begin{array}{l}\text { Aquí se detallan las competencias que el estu- } \\
\text { diante adquiere en su proceso de aprendizaje, se } \\
\text { encuentra dentro del protocolo del curso. }\end{array}$ \\
\hline Análisis de los estudiantes y del contexto & $\begin{array}{l}\text { La UNAD es una universidad abierta dentro de su } \\
\text { misión está la educación para todos, Dentro } \\
\text { del CORE se encuentra actividades de fase } \\
\text { de reconocimiento que busca determinar los co- } \\
\text { nocimiento previos del estudiantes }\end{array}$ \\
\hline Redacción de los objetivos & $\begin{array}{l}\text { Las mentas de aprendizaje determinan señala- } \\
\text { mientos específicos que los estudiantes podrán } \\
\text { hacer al final de la instrucción, se encuentra den- } \\
\text { tro del protocolo del curso. }\end{array}$ \\
\hline Desarrollo de instrumentos de evaluación & $\begin{array}{l}\text { A través de las diferentes actividades que se } \\
\text { encuentran en las fases de reconocimiento, } \\
\text { profundización y transferencia. }\end{array}$ \\
\hline Elaboración de la estrategia instruccional & $\begin{array}{l}\text { La UNAD hace uso del trabajo colaborativo como } \\
\text { estrategia instruccional, en el los estudiantes } \\
\text { desarrollan actividades en grupo, adquieren ade- } \\
\text { más de conocimiento valores y habilidades del } \\
\text { trabajo en equipo. }\end{array}$ \\
\hline
\end{tabular}




\begin{tabular}{|l|l|}
\hline MODELO DICK, CAREY Y CAREY & ESTÁNDAR CORE UNAD CAREY \\
\hline $\begin{array}{l}\text { Desarrollo y selección de materiales } \\
\text { de instrucción }\end{array}$ & $\begin{array}{l}\text { En el CORE se encuentran contenidos en texto y } \\
\text { Objetos Virtuales de Aprendizaje OVAS los cua- } \\
\text { les son significativos en la educación en línea. }\end{array}$ \\
\hline $\begin{array}{l}\text { Diseño y desarrollo de la } \\
\text { evaluación formativa }\end{array}$ & $\begin{array}{l}\text { Aunque la evaluación formativa puede darse } \\
\text { uno a uno o en grupo, no se presenta dentro del } \\
\text { CORE un espacio para evaluar, los materiales o } \\
\text { la instrucción. }\end{array}$ \\
\hline $\begin{array}{l}\text { Diseño y desarrollo de la } \\
\text { evaluación sumativa }\end{array}$ & $\begin{array}{l}\text { Tampoco existe dentro del CORE un instrumento } \\
\text { que permita valorar la instrucción. }\end{array}$ \\
\hline Revisión de la instrucción & $\begin{array}{l}\text { Quienes diseñan las actividades y materiales } \\
\text { dentro de un curso son los directores de curso, } \\
\text { ellos son quienes deben recoger información } \\
\text { para determinar si esta debe ser mejorada, pero } \\
\text { teniendo en cuenta que no se presenta evalua- } \\
\text { ción formativa, no es claro si se presenta o no } \\
\text { una revisión de la instrucción }\end{array}$ \\
\hline
\end{tabular}

La tabla 2 establece un paralelo entre el CORE y el modelo de diseño instruccional de Dick, Carey y Carey se puede observar que existen algunos elementos de este modelo dentro del CORE.

\section{Conclusiones}

La orientación pedagógica de la Universidad está encaminada al aprovechamiento de las inmensas posibilidades de aplicación que ofrecen las Tecnologías de la Información y de las Comunicaciones (TIC). Es así como la UNAD se proyecta para transformarse en una organización flexible y dispuesta a la virtualidad, que garantice el acceso a la formación de todas las personas que quieran educarse y brindar el mayor número de medios posibles manteniendo su identidad cultural, tradicional e histórica como organización educativa Abierta y a Distancia.

Para apoyar el auto aprendizaje la UNAD está realizando grandes esfuerzos en ofrecer las nuevas tecnologías de la información y de la comunicación al servicio de sus docentes y estudiantes. Para tal fin se ha dotado con la infraestructura tecnológica necesaria para incrementar los niveles de conectividad. Sin embargo, es muy prolífero el horizonte sobre el cual se puede desarrollar este gran proceso, es decir, las herramientas informáticas que proporcionen el objetivo deseado.

El CORE es considerado el dispositivo académico, pedagógico, curricular y didáctico mediante el cual se diseña, virtualiza y ofrece el conjunto de cursos académicos que sirven como base para el desarrollo de los procesos formativos que adelanta la institución. Estoya que no existe un modelo instruccional 
definido que permita establecer claramente la estructura instruccional de los cursos ofertados por la Universidad.

Algunos cursos como el de competencias comunicativas considera al modelo ASSURE en algunos de los procesos de construcción del curso en línea. Sin duda, otro valor agregado a una propuesta bajo el modelo ASSURE garantiza que las actividades en su totalidad están pensadas y orientadas en función del aprendizaje desde un paradigma constructivista. De acuerdo con el análisis de los pasos del modelo ASSURE y su aplicación a la educación a distancia, es importante considerar que representa una oportunidad de innovación para un profesor. Este podrá diseñar su curso siguiendo las etapas de este modelo, yle permitirá incursionar en forma efectiva en un curso a distancia -sobre todo si es un instructor novato en estos ambientes de aprendizaje-.

De acuerdo con el comparativo realizado entre el estándar CORE y el modelo del Diseño Instruccional de Dick, Carey y Carey, se puede decir que el CORE posee algunos elementos de este modelo pero no es un modelo de Diseño Instruccional en sí. La estructura de los cursos virtuales poseen siete de las 10 fases del modelo de Dick, Carey y Carey, lo que corresponde a la evaluación formativa y sumativa pero no se evidencia, la revisión de la instrucción; elementos muy importante para determinar las mejoras o cambios que se deben hacer en el curso y para identificar si la instrucción cumplió con sus objetivos.

La UNAD desde el 2005 ha venido incursionando en la modalidad de educación en línea donde las actividades son el punto fundamental del proceso formativo. Por ejemplo, el video de la conferencia Arqueología de la Educación a Distancia en la UNAD menciona el modelo instruccional e-learning. Sin embargo no hay una teoría que demuestre este modelo, ya que el e-learning es una modalidad educativa.

El Diseño Instruccional se considera parte central de todo proceso de aprendizaje porque representa la planificación detallada de las actividades educativas sin importar la modalidad de enseñanza. Representa un proceso fundamentado en teorías de disciplinas académicas, especialmente en las disciplinas relativas al aprendizaje humano, por lo tanto se hace necesario que la UNAD defina un modelo instruccional unificado para todos los programas. Este modelo puede ser modelo de Diseño Instruccional de Dick, Carey y Carey o el modelo de ASSURE.

\section{Referencias bibliográficas}

Anglin, G. y G. Morrison. 2000. Technology Trends and Issues: Readings. Fort Lauderdale: Nova Southeastern University. 
Ávila Baray, H. L. 2006. Introducción a la metodología de la investigación [versión electrónica]. Tomado --- de www.eumed.net/libros/2006c/203/

Bavaresco de Pietro, A.M. 1997. Proceso metodológico en la investigación (Cómo hacer un diseño de investigación). Maracaibo: Universidad del Zulia.

Benítez Lima, M. G. 2010. «Modelo de diseño instruccional ASSURE aplicado a la educación a distancia». Tlatemoani. Revista académica de investigación. 1. Tomado el 16 de febrero de 2011 de http://www.eumed.net/rev/tlatemoani/01/mgbl.htm

Bunge, M. 1985. La investigación cientifica. Barcelona: Ariel.

Casas Armengol, M. y L. Stojanovic de Casas. 2004. «Virtualización de la Universidad Latinoamericana». Informe de Investigaciones Educativas. 18: 15-24.

Chiappe Laverde, A. 2006. Modelo de Diseño Instruccional basado en Objetos de Aprendizaje (MDIBOA): Aspectos relevantes [versión electrónica]. Tomado el --- de http://oas.unisabana.edu.co/files/MDIBOA.pdf

García Aretio, L. 2002. "La Cátedra Unesco de Educación a Distancia (CUED) de la UNED de España. Una apuesta por la calidad de la Educación a Distancia». Universidades. 24: 11-17.

Gil, M. C. 2004. «Modelos de Diseño Instruccional para Programas Educativos a Distancia». Perfiles Educativos. 24: 93-114.

Heinich, R. et al. 1999. Instructional Media and Technologies for Learning. New Jersey: Prentice-Hall.

Martínez, A. C. 2009. «El Diseño Instruccional en la Educación a Distancia. Un acercamiento a los modelos». Apertura. 9 (10): 104-119.

Morales, C. 2006. «La importancia del Diseño Instruccional en el diseño de cursos en línea». Didáctica Innovación y Multimedia. 3. Tomado el 16 de febrero de $2011 \mathrm{de}$ http://dim.pangea.org/revistaDIM3/Articulos/CMorales.pdf

Muñoz, P. 2010. «Modelo de diseño instruccional utilizado en ambientes teleinformáticos». Revista de Investigación Educativa ConeCT@2 .1(2). Tomado el 16 de febrero de 2011 de http://www.revistaconecta2.com.mx/2modelos.pdf

Ortiz, J. y J. Prato. 2004. «Moodle: Gestor de cursos en línea». Informe de Investigaciones Educativas. 18: 95-96.

Salazar, R. et al. 2008. Curso de Diseño y Administración de Aulas Virtuales en el Campus Virtual de la UNAD. Bogotá: GIT- Universidad Nacional Abierta y a Distancia. 
Sampieri, R. et al. 1998. Metodología de la investigación. México: Mc. Graw-Hill.

Simonson, M. et al. 2006. Teaching and Learning at a Distance: Foundations Of Distance Education. Upper Saddle River, NJ: Pearson Prentice Hall

Suárez, N. 1999. La investigación documental paso a paso. Mérida: Consejo de publicaciones de la Universidad de los Andes.

\section{Anexos}

Cuadro 1: Elementos del curso CORE

\begin{tabular}{|c|}
\hline Nombre del curso \\
\hline $\begin{array}{l}\text { Tópico cero } \\
\text { * Noticias del aula } \\
\text { * Foro General del curso } \\
\text { * Agenda } \\
\text { * Portafolio de grupo wiki } \\
\text { * Glosario }\end{array}$ \\
\hline $\begin{array}{l}\text { Tópico uno } \\
\text { Contenido } \\
\text { * Protocolo Académico del curso } \\
\text { * Desarrollo Unidades temáticas (Módulo) }\end{array}$ \\
\hline $\begin{array}{l}\text { Tópico dos } \\
\text { Fase de reconocimiento } \\
{ }^{*} \text { Revisión de pre saberes (Lección evaluativa) } \\
{ }^{*} \text { Reconocimiento del curso (Foro y tarea) }\end{array}$ \\
\hline
\end{tabular}

Tópico tres

Actividades unidades: unidad 1

${ }^{*}$ Reconocimiento Unidad 1 (Lección evaluativa)

* Trabajo colaborativo Unidad 1 (Foro y tarea)

* Lección Evaluativa Unidad 1

${ }^{*}$ Quiz Unidad 1

Cada una de las unidades tendrá estos mismos elementos

Tópico cuatro

Prueba final 